\section{Vulnerabilidade ambiental, social e viária em acidentes com transporte de produtos perigosos: estudo de caso na BR-101 entre Osório e Torres, Rio Grande do Sul, Brasil}

\author{
Environmental, social, and roadway vulnerability \\ in accidents involving transportation of hazardous \\ products: a case study of the BR-101 highway \\ between Osório and Torres in Rio Grande do Sul \\ State, Brazil
}

\author{
Vulnerabilidad ambiental, social y vial en \\ accidentes con transporte de productos \\ peligrosos: estudio de caso en la BR-101 entre \\ Osório y Torres, Río Grande do Sul, Brasil
}

Maria Auxiliadora Cannarozzo Tinoco ${ }^{1}$ Christine Tessele Nodari 1 Kimberllyn Rosa da Silva Pereira 1

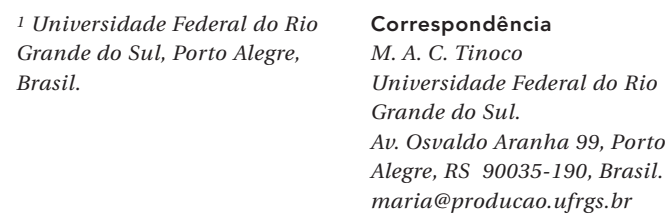

Correspondência M. A. C. Tinoco Universidade Federal do Rio Grande do Sul.

Av. Osvaldo Aranha 99, Porto Alegre, RS 90035-190, Brasil. maria@producao.ufrgs.br 


\section{Introdução}

No Brasil, o transporte de produtos perigosos é realizado, principalmente, através do modal rodoviário, como consequência da estrutura da matriz de transportes do país 1,2. A preocupação com os acidentes envolvendo produtos perigosos no modal rodoviário vem crescendo cada vez mais, segundo a Companhia Ambiental do Estado de São Paulo (CETESB) ${ }^{3}$, devido ao prejuízo ambiental e social decorrente de acidentes dessa natureza, constituindo um problema de saúde pública 4,5 .

Uma vez que a atividade de transporte de produtos perigosos pode resultar em acidentes envolvendo riscos para a saúde de pessoas, para segurança pública e para o meio ambiente, trata-se de uma atividade fortemente regulamentada. Assim, é submetida às regras e aos procedimentos estabelecidos no Regulamento para o Transporte Rodoviário de Produtos Perigosos 6 da Agência Nacional de Transportes Terrestres (ANTT). Dentre as principais normas específicas para esse tipo de transporte estão as Resoluções ANTT no 3.886/12 7 e ANTT no 3.665/11 8, contendo alterações no Regulamento do Transporte Rodoviário de Produtos Perigosos, e a Resolução ANTT no 420/04 9, que estabelece as Instruções Complementares ao Regulamento e a conceituação legal de produtos perigosos para fins de transporte. Essas normas visam a propiciar que a operação do transporte desse tipo de produto ocorra dento de níveis adequados de segurança, para evitar os impactos sociais e ambientais decorrentes de possíveis acidentes viários.

A preocupação com esses impactos tem dado origem a diversos estudos de análises de risco e de vulnerabilidade ambiental e social, que podem nortear ações corretivas e preventivas para reduzir ou minimizar o impacto dos acidentes envolvendo o transporte rodoviário de produtos perigosos. Alguns estudos apresentam uma ênfase na vulnerabilidade social 10,11 , enquanto outros abordam a vulnerabilidade social e ambiental 2,12,13. Outros autores realizam a análise de vulnerabilidade por meio de ferramentas multicritério, envolvendo não somente os aspectos ambientais e sociais, mas também a análise das características relacionadas à rodovia 3,14 . Nesses estudos, a vulnerabilidade é definida como parte essencial do risco e refere-se à susceptibilidade social e ambiental a uma situação de perigo, neste caso, gerada pela ocorrência de acidentes com produtos perigosos 14 .

No contexto brasileiro, estudos sobre vulnerabilidade e riscos associados ao transporte rodoviário de produtos perigosos são escassos 15 . Um dos trabalhos que lideram as pesquisas bra- sileiras sobre metodologias para o gerenciamento dos riscos no transporte de produtos perigosos é o Programa de Gerenciamento de Riscos para Administradores de Rodovias para o Transporte de Produtos Perigosos (PGR) 3. O PGR da CETESB propõe a avaliação da vulnerabilidade nos aspectos ambiental (vegetação e recursos hídricos) e social, o levantamento dos trechos de maior incidência de acidentes e, com base nestas informações, a identificação de trechos críticos de maior vulnerabilidade na ocorrência de acidentes com produtos perigosos.

Um dos fatores observados nos trabalhos sobre vulnerabilidade e riscos no transporte de produtos perigosos, que incorporam a análise da rodovia, é que esta análise é realizada em termos de probabilidade de ocorrência de acidentes, baseada em dados históricos. Porém, sabe-se que os acidentes observados em uma via são eventos altamente influenciados pelo acaso. Dessa forma, são necessários períodos longos de registros de acidentes para que pontos críticos (locais onde há concentração de acidentes decorrentes de causas especificas, não devido ao acaso) sejam identificados com razoável nível de significância. Quando não é possível obter dados adequados à análise reativa da segurança (baseada em dados observados de acidentes) é necessária a adoção de uma abordagem proativa baseada em auditoria de segurança viária. O cenário do presente estudo não permitiu a obtenção de dados históricos de acidentes, por constituir um trecho rodoviário recentemente remodelado com mudança da classe da rodovia de pista simples para pista dupla e com redefinição de traçado em determinados segmentos, exigindo a adoção de uma abordagem proativa baseada em auditoria de segurança viária.

Nesse contexto, o presente trabalho objetiva a avaliação da vulnerabilidade ambiental e social, na ocorrência de acidentes com transporte de produtos perigosos na rodovia BR-101, entre os municípios de Osório e Torres no Estado do Rio Grande do Sul. O trecho sul da rodovia BR101, que vai de Torres a Osório, constitui uma das principais rodovias federais do Brasil, servindo de ligação para outros países do Mercosul e, por este motivo, tem um grande tráfego de produtos perigosos. Devido à preocupação com os acidentes envolvendo produtos perigosos nessa rodovia, surge a necessidade de realizar uma análise de vulnerabilidade multicriterial que permita avaliar o risco potencial da rodovia e os aspectos de sensibilidade ambiental e social na ocorrência de acidentes com produtos perigosos.

Portanto, pretende-se com o estudo identificar os trechos críticos de maior vulnerabilidade na rodovia em questão, baseando-se na 
proposição e aplicação de uma abordagem que integra a análise de sensibilidade dos aspectos ambientais e sociais, adaptada do programa proposto pela CETESB e, a análise de segurança viária da rodovia pesquisada, baseada no método de Auditoria Assistida proposto por Nodari 16. Os resultados gerados poderão ser de utilidade na geração de mapas para o gerenciamento de riscos e no Plano de Ação Emergencial da Rodovia BR-101.

Finalmente, a pesquisa visa a contribuir à literatura científica a partir da consolidação de um método para estimar a vulnerabilidade na ocorrência de acidentes com o transporte rodoviário de produtos perigosos, que considere a sensibilidade dos elementos ambientais (hídricos e vegetação) e sociais (ocupação humana), assim como o risco viário imposto pela rodovia, preenchendo lacunas da literatura apontadas anteriormente.

\section{Métodos}

A abordagem metodológica de análise da vulnerabilidade ambiental e social associada ao transporte de produtos químicos perigosos, proposta e aplicada neste estudo, está fundamentada na avaliação de aspectos físicos de segurança viária e na determinação da sensibilidade de aspectos ambientais e sociais na ocorrência de possíveis acidentes com produtos químicos perigosos, na área de influência da rodovia BR-101 em uma extensão de $88 \mathrm{~km}$ entre os municípios de Torres e Osório. Foi definida como área de influência para a análise da vulnerabilidade uma distância de até $2 \mathrm{~km}$ a partir dos bordos da pista, conforme recomendações do Departamento Nacional de Infraestrutura de Transportes (DNIT) 1. Os procedimentos utilizados envolvem pesquisa bibliográfica, observação direta do local (pesquisas de campo realizadas nos dias 26 de junho e 2 de outubro de 2014) e pesquisa documental, por meio de análise de mapas. O método de trabalho foi estruturado nas seguintes etapas: (i) auditoria de segurança viária; (ii) avaliação da sensibilidade ambiental e social; (iii) determinação do índice de vulnerabilidade; e (iv) geração de trechos críticos.

\section{Auditoria de segurança viária}

A auditoria de segurança viária no segmento da rodovia em estudo baseou-se no modelo proposto por Nodari 16 para a avaliação do risco viário potencial de segmentos rodoviários. Por meio de um procedimento padronizado de coleta de dados em campo foram obtidas notas, quilômetro a quilômetro, para 34 características consideradas no método. Os valores do índice de Risco Viário (RV) foram obtidos baseando-se nos pesos estimados para as características viárias e nas notas atribuídas nas inspeções em campo. O método, adotado originalmente na avaliação de rodovias de pistas simples, é aplicável à rodovia estudada visto que inclui a avaliação de caraterísticas viárias comuns aos ambientes de pista simples e pista dupla. No entanto, na rodovia em estudo esperam-se notas atribuídas nas inspeções superiores ao padrão encontrado em rodovias simples, o que caracteriza o melhor potencial de segurança desta classe de rodovia.

As características viárias consideradas no método estão organizadas em nove macrocategorias. São elas: superfície do pavimento; curvas; interseções/acessos; sinalizações vertical e horizontal; elementos longitudinais; elementos da seção transversal; usuários vulneráveis; laterais da via; e elementos gerais.

A formulação do índice de RV consiste no somatório do produto entre o peso e a nota das características dentro de cada macrocategoria (equação 1). Os pesos representam o grau de influência potencial de cada caraterística da rodovia na ocorrência de acidentes. As notas das características representam o estado vigente de cada característica no período da análise. O $\mathrm{RV}_{\mathrm{sj}}$ referente a cada uma das macrocategorias constitui um modelo compensatório, em que a presença de uma característica em nível alto compensa a presença de outra característica em nível baixo.

$R V_{S j}=\sum_{i=1}^{m}\left(p_{i, j} \times n_{i, j}\right)$

onde, $\mathrm{RV}_{\mathrm{sj}}$ : RV da macrocategoria j para um segmento de $1 \mathrm{~km}$ de extensão; $\mathrm{n}_{\mathrm{i}, \mathrm{j}}$ : nota da característica i resultante da inspeção em campo; e m: quantidade de características dentro da macrocategoria j.

A consolidação do RV das nove macrocategorias para a obtenção do índice de risco viário potencial geral, de cada segmento de um quilômetro avaliado $\left(\mathrm{RV}_{\mathrm{Gk}}\right)$, é obtida segundo a média geométrica dos $R_{\mathrm{j}}$ parciais de cada macrocategoria. Diferentemente da média aritmética, a média geométrica caracteriza-se por penalizar a variabilidade dos valores utilizados na obtenção da média. Seu uso é, normalmente, indicado para os casos de qualidades não compensatórias. $\mathrm{O}$ uso da média geométrica é apropriado uma vez que o mau desempenho em uma macrocategoria (por exemplo, sinalização) não é compensado pelo bom desempenho em outra macrocategoria (por exemplo, interseções/acessos). $\mathrm{O} \mathrm{RV}_{\mathrm{Gk}}$ é obtido com base na equação 2 . 


$$
R V_{G k}=\sqrt[9]{R V_{\text {sup }}+R V_{\text {cur }}+R V_{\text {int }}+R V_{\text {sin }}+R V_{\text {lon }}+R V_{\text {tran }}+R V_{\text {vul }}+R V_{\text {lat }}+R V_{\text {el.g }}}
$$

em que, $\mathrm{RV}_{\mathrm{Gk}}$ : $\mathrm{RV}$ global do segmento $\mathrm{k}$; $\mathrm{RV}_{\text {sup }}$ : RV da macrocategoria "superfície do pavimento"; $\mathrm{RV}_{\text {cur }}: \mathrm{RV}$ da macrocategoria "curvas"; $\mathrm{RV}_{\text {int }}: \mathrm{RV}$ da macrocategoria "interseções/acessos"; $\mathrm{RV}_{\text {sin }}$ : RV da macrocategoria "sinalização vertical e horizontal”; $\mathrm{RV}_{\text {lon: }} \mathrm{RV}$ da macrocategoria "elementos longitudinais"; $\mathrm{RV}_{\text {tran: }}$ RV da macrocategoria "elementos da seção transversal"; $\mathrm{RV}_{\mathrm{vul}}: \mathrm{RV}$ da macrocategoria "usuários vulneráveis"; $\mathrm{RV}_{\text {lat: }}$ RV da macrocategoria "laterais da via"; $\mathrm{RV}_{\text {el.g: }} \mathrm{RV}$ da macrocategoria "elementos gerais"; e K: segmentos rodoviários de $1 \mathrm{~km}$ de extensão.

Por fim, a condição de risco viário potencial de todo o trecho rodoviário avaliado (conjunto de vários segmentos de $1 \mathrm{~km}$ ) é indicada pelo RV, que é obtido pela média geométrica dos $\mathrm{RV}_{\mathrm{Gk}} \mathrm{de}$ cada quilômetro avaliado. O uso da média geométrica para obtenção dos RV tem por objetivo privilegiar os trechos rodoviários que apresentam segmentos mais homogêneos quanto às condições de segurança. Dessa forma, busca-se minimizar a indesejável violação da expectativa dos motoristas que ocorre quando estes são submetidos a trechos rodoviários que alternam segmentos com boas e más condições de segurança. O RV é obtido baseando-se na equação 3.

$$
R V=\sqrt[n]{\prod R V_{G k}}
$$

em que, RV: RV do trecho avaliado (composto por $\mathrm{n}$ segmentos) e n: número de segmentos de $1 \mathrm{~km}$ que compõem o trecho avaliado.

O RV geral do trecho é indicado para análises do trecho de rodovia como um todo. Os índices referentes aos segmentos, por sua vez, são indicados para orientar decisões específicas sobre os locais a tratar e tipos de intervenções a adotar, uma vez que indicam os segmentos que apresentam maiores deficiências de segurança $\left(R_{G k}\right)$ e as macrocategorias que estão mais deficientes $\left(R_{j}\right)$. Ainda é possível, por meio da análise nas notas das inspeções de campo, verificar qual a característica dentro das macrocategorias que apresenta os maiores problemas quanto à segurança.

A adoção de um procedimento padronizado de coleta de dados em campo consiste no uso do gabarito de notas associado a uma escala semântica, para a avaliação das condições vigentes na rodovia das 34 características constantes no RV. Dessa forma, busca-se reduzir a variabilidade resultante de inspeções realizadas por diferentes profissionais, a fim de permitir a comparabilidade dos índices obtidos em diferentes pontos do tempo e do espaço.

\section{Avaliação da sensibilidade ambiental e social}

A avaliação da sensibilidade ambiental e social no trecho em estudo consistiu no levantamento e ponderação da sensibilidade dos elementos de vulnerabilidade (vegetação, recursos hídricos e ocupação humana) durante as saídas de campo e na validação desta avaliação baseando-se na análise de mapas. A avaliação da sensibilidade ambiental e social foi fundamentada na metodologia proposta pelo PGR da CETESB ${ }^{3}$ e de estudos propostos por Bubbico et al. ${ }^{11}$ e Souza et al. ${ }^{2}$, para a análise de riscos no transporte rodoviário de produtos perigosos.

Inicialmente, foram atribuídas estimativas de sensibilidade com base na observação realizada dos aspectos da vegetação, recursos hídricos e ocupação humana na área de influência da rodovia ( $2 \mathrm{~km}$ a partir da borda da pista), a cada quilômetro do trecho em estudo e nos dois sentidos da rodovia (norte e sul). As observações foram realizadas separadamente, uma vez que trata-se de um trecho de pista dupla onde a área de influência a partir dos bordos da pista apresenta elementos de vulnerabilidade diferentes nos dois sentidos. Para a ponderação da sensibilidade nos aspectos de vulnerabilidade utilizaram-se escalas conforme a criticidade e particularidade de cada categoria.

A sensibilidade da vegetação foi atribuída valendo-se da análise do tipo de cobertura vegetal e da existência de Unidade de Proteção ou Área de Proteção Ambiental na área de influência, conforme critérios utilizados no Código Florestal vigente (Lei no 9.985/2000 17) e categorização da vegetação de acordo com o Mapa de Vegetação do Brasil 18. A ponderação da sensibilidade foi realizada usando-se a seguinte escala:

- Presença de Unidade de Proteção: sensibilidade crítica (ponderação de 9 a 10);

- Áreas de serra e floresta densa de mata nativa: sensibilidade elevada (ponderação de 7 a 9);

- Áreas de formações pioneiras: sensibilidade moderada (ponderação de 3 a 7);

- Áreas alagadas, banhados, formações pioneiras fora da área de influência: sensibilidade baixa (ponderação de 0 a 3).

A classificação da sensibilidade dos recursos hídricos em cada trecho de análise considerou: a classificação dos recursos hídricos conforme a Resolução CONAMA no 357 de 14 de março de 2005 19; a proximidade com a rodovia e a existência de captação pública até uma distância de $5 \mathrm{~km}$. A classificação da sensibilidade dos recursos hídricos avaliados a cada quilômetro da rodovia foi atribuída com base na seguinte escala: 
- Pontes sobre rio e margens de lagoa (até 100m) para águas classes especial 1, 2 e 3: sensibilidade crítica (ponderação de 9 a 10);

- Águas classes especial 1, 2 e 3 até $2 \mathrm{~km}$ da rodovia com captação pública até $5 \mathrm{~km}$ : sensibilidade elevada (ponderação de 5 a 9);

- Águas classes especial 1, 2 e 3 fora da área de influência com captação pública até $5 \mathrm{~km}$ : sensibilidade moderada (ponderação de 2 a 5);

- Águas classes especial 1, 2 e 3 fora da área de influência sem captação pública até $5 \mathrm{~km}$ da rodovia: sensibilidade baixa (ponderação de 0 a 2). A sensibilidade da ocupação humana no trecho em estudo foi fundamentada em trabalhos da literatura 3,10,11 e considerou a estimativa do percentual de cobertura da ocupação humana a cada quilômetro analisado. Dessa forma, a escala de sensibilidade da ocupação humana na área de influência da rodovia é a seguinte:

- Urbana: ocupação humana com cobertura de assentamentos maior que $70 \%$ da área do trecho analisado: sensibilidade crítica (ponderação de 9 a 10);

- Suburbana: ocupação humana com cobertura de assentamentos entre $40 \%$ e $70 \%$ da área do trecho analisado: sensibilidade elevada (ponderação de 5 a 9);

- Rural: ocupação humana com cobertura de assentamentos entre $10 \%$ e $40 \%$ da área do trecho analisado: sensibilidade moderada (ponderação de 2 a 5);

- Remota: ocupação humana com cobertura de assentamentos menor que $10 \%$ da área do trecho analisado: sensibilidade baixa (ponderação de 0 a 2).

As estimativas de sensibilidade ambiental e social realizadas durante as saídas de campo foram validadas pela aplicação da técnica da quadrícula em mapas. A técnica consistiu na sobreposição de duas quadrículas de $1 \mathrm{~km}^{2}$ de área, constituídas de 100 quadrados cada uma, no mapa da região contendo o trecho da rodovia em estudo e a delimitação dos quilômetros avaliados e da área de influência da rodovia. A cada quilômetro analisado na área de influência da rodovia $(2 \mathrm{~km})$ foram aplicadas as quadrículas e calculados os percentuais de cobertura da vegetação, dos recursos hídricos e da ocupação humana. De acordo com o percentual de cobertura foi atribuído um valor de sensibilidade (de 0 a 10) em cada um dos aspectos avaliados.

O mapa da região foi gerado no Laboratório de Geoprocessamento (Centro de Ecologia, Instituto de Biociências, Universidade Federal do Rio Grande do Sul), com base na coleta de coordenadas a cada quilômetro durante as saídas de campo e com a ajuda do sistema WGS84. A aplicação da quadrícula foi realizada por meio da utilização do software Google Earth, disponível na Internet (https://www.google.com/earth/).

A sensibilidade final relativa a cada um dos aspectos de vulnerabilidade em cada trecho analisado foi resultado da média dos valores de sensibilidade atribuídos nas duas saídas de campo e da análise das quadrículas, sendo que os valores obtidos de sensibilidade da primeira quadrícula (de 0 a $1 \mathrm{~km}$ da rodovia) tiveram um maior peso no cálculo da média, em relação aos valores de sensibilidade da segunda quadrícula (de 1 a $2 \mathrm{~km}$ ), pois o impacto de acidentes com produtos perigosos será maior nos aspectos ambientais e sociais próximos da rodovia.

\section{Determinação do índice de vulnerabilidade}

Com base nos valores médios de sensibilidade para cada aspecto ambiental e social, e considerando o RV obtido pela auditoria de segurança viária, foi estimado um índice de vulnerabilidade a cada quilômetro analisado. O índice proposto buscou refletir a vulnerabilidade de cada segmento da rodovia quanto à ocorrência de acidentes com produtos perigosos. $\mathrm{O}$ índice de vulnerabilidade foi determinado pela equação 4 :

$V=4+\left(\left(S_{H i} \times 0,25\right)+\left(S_{V e} \times 0,25\right)+\right.$

$\left.\left(S_{H u} \times 0,3\right)+\left(R_{V} \times 0,2\right)\right)$

em que, V: índice de vulnerabilidade; $\mathrm{S}_{\mathrm{Hi}}$ : sensibilidade média dos recursos hídricos; $\mathrm{S}_{\mathrm{Ve}}$ : sensibilidade média da vegetação; $\mathrm{S}_{\mathrm{Hu}}$ : sensibilidade média da ocupação humana; RV: risco viário.

Os pesos que ponderam os valores de sensibilidade na equação 4 estão fundamentados na importância atribuída aos aspectos sociais e ambientais na ocorrência de acidentes com produtos perigosos, conforme recomendações do DNIT 1, em que o impacto de acidentes com produtos perigosos é maior para a população adjacente ao local e, logo, para os recursos hídricos e vegetação.

A adoção da constante da equação leva em consideração que mesmo uma rodovia perfeita no seu componente viário ambiental e com níveis baixos de sensibilidade está sujeita à ocorrência de acidentes viários, e que os resíduos deste acidente terão um impacto sobre esta localidade, afetando seu ambiente natural. A esse impacto foi atribuído o valor base igual a 4, que representa uma baixa vulnerabilidade. A introdução dessa constante é uma decisão conservadora que indica que a simples presença de uma rodovia em um sistema natural qualquer impõe uma vulnerabilidade que, mesmo pequena, não deve ser negligenciada.

Sabe-se que o risco de ocorrência de acidentes viários em um determinado ponto da rede 
é função de um conjunto de variáveis conhecidas como fatores contribuintes, tradicionalmente agrupados em três componentes do sistema de tráfego: componente veicular, componente viário-ambiental e componente humano 20. Estima-se que falhas em fatores do componente viário ambiental possam influenciar a gênese de aproximadamente $30 \%$ dos acidentes 21 . No entanto, Ogden 22 enfatiza que os fatores do componente viário ambiental (por exemplo, pavimento, sinalização, perfil transversal) têm forte influência sobre o desempenho de fatores relativos aos componentes humano e veicular. Sendo, portanto, um elemento chave na determinação do real risco viário. Assim, os elementos físicos e geométricos de uma rodovia podem impactar no risco viário em uma proporção ainda maior do que os porcentuais estimados com base nos dados de acidentes viários observados. Essas considerações subsidiaram a escolha do valor da constante para representar o valor base do índice de vulnerabilidade.

Dessa forma, obteve-se um índice de vulnerabilidade a cada quilômetro analisado em cada uma das pistas da rodovia. O índice de vulnerabilidade final de cada trecho (cada quilômetro analisado) foi determinado pelo maior valor obtido em cada um dos sentidos da rodovia.

\section{Geração de trechos críticos}

Baseando-se nos valores de índice de vulnerabilidade foram determinados os trechos mais críticos da rodovia. Esses trechos correspondem àqueles de valores de vulnerabilidade acima de 8,5 . Porém, os trechos com vulnerabilidade elevada (entre 7,5 e 8,4) também merecem atenção no sentido da prevenção e da ação emergencial no caso da ocorrência de acidentes. Portanto, nos trechos considerados de vulnerabilidades elevada e crítica, devem ser indicadas medidas para minimizar a ocorrência de acidentes com produtos perigosos e os efeitos gerados ao meio ambiente e à população.

\section{Resultados da análise de vulnerabilidade}

\section{Risco viário}

As Figuras 1 e 2 apresentam os gráficos resultantes das análises de segurança viária e de sensibilidade ambiental e social (recursos hídricos, vegetação e ocupação humana) do trecho em estudo, no sentido norte e sul, respectivamente.

Como observado nas Figuras 1 e 2, o RV oscila entre 1 e 3 . Esses valores indicam a existência de baixo RV potencial, visto que se trata de uma rodovia de pista dupla recentemente concluída Os principais elementos que induzem risco no trecho avaliado estão associados a deficiências na provisão de travessias de pedestres seguras e à declividade dos taludes laterais. Destaca-se também que a oscilação do nível de RV ao longo do trecho estudado é, por si só, um elemento que induz risco ao trecho. Esse fato decorre da violação da expectativa do motorista, que se forma pela leitura que o mesmo faz das condições oferecidas pela via nos trechos já percorridos. $\mathrm{O}$ motorista então assume que esse padrão viário será mantido. Quando ocorrem situações viárias que diferem da esperada, o motorista pode não responder adequadamente a esse novo ambiente 23 .

\section{Análise de sensibilidade ambiental e social}

$\mathrm{Na}$ análise de sensibilidade da vegetação no sentido norte da rodovia (Figura 1), observouse um ponto de maior sensibilidade ambiental corresponde à área onde está localizada a Área de Proteção Ambiental (APA) Municipal de Terra de Areia ( $\mathrm{km} \mathrm{43).} \mathrm{A} \mathrm{cobertura} \mathrm{vegetal} \mathrm{de} \mathrm{grande}$ parte do trecho no sentido norte da rodovia é caracterizada por planícies e formações pioneiras de menor sensibilidade, porém, identificou-se em alguns pontos a presença de formações nativas densas (floresta ombrófila densa), onde a sensibilidade ambiental é elevada na ocorrência de acidentes com produtos perigosos na rodovia (por exemplo, km 3 a 5; km 62 a 65; km 69).

A sensibilidade da vegetação no sentido sul da rodovia (Figura 2), de forma geral, é maior do que no sentido norte. Isso devido ao tipo de cobertura vegetal predominante (áreas de serra, formações nativas densas), característico da floresta ombrófila densa. O ponto de maior sensibilidade ambiental acontece nos quilômetros 84 a 88 devido à presença da APA Municipal de Osório (Morro da Borússia).

Em relação à sensibilidade dos recursos hídricos, observou-se que os maiores valores de sensibilidade acontecem nos quilômetros da rodovia onde estão localizadas as pontes sobre rio (Rio Cardoso no km 21, Rio Chimarrão no km 31, Rio Três Forquilhas no km 40, Rio Sanga Funda no km 53 e Rio Maquiné no km 65), os pontos de

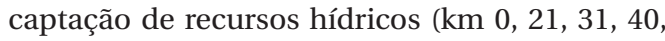
53, 65 e 76) e nas margens das lagoas (Lagoa Itapeva do km 10 ao 20 e km 29, Lagoa dos Quadros do km 54 ao 61, Lagoa da Pinguela do km 76 ao 78 e Lagoa do Peixoto no km 84). A presença desses sistemas lagunares acontece no sentido norte da rodovia, como pode ser observado na Figura 1.

Em relação à ocupação humana, verifica-se que os pontos de maior sensibilidade na área de influência da rodovia correspondem às regiões 
1a) Risco viário

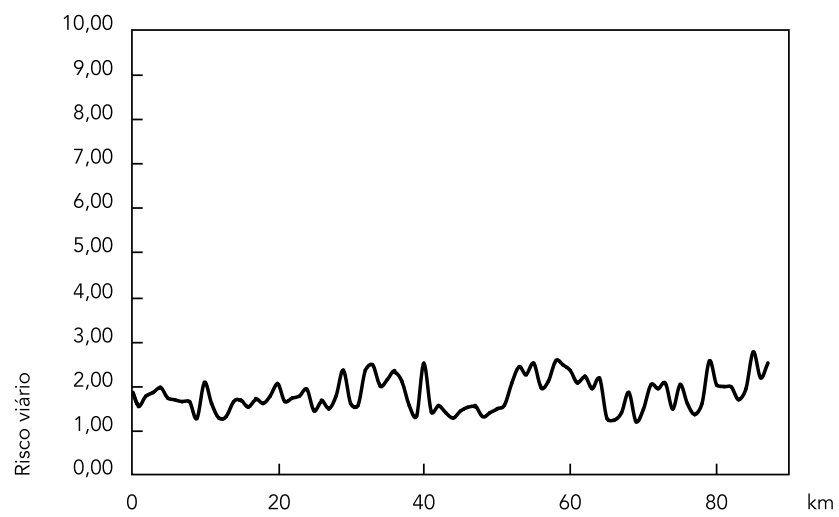

1c) Sensibilidade e recursos hídricos

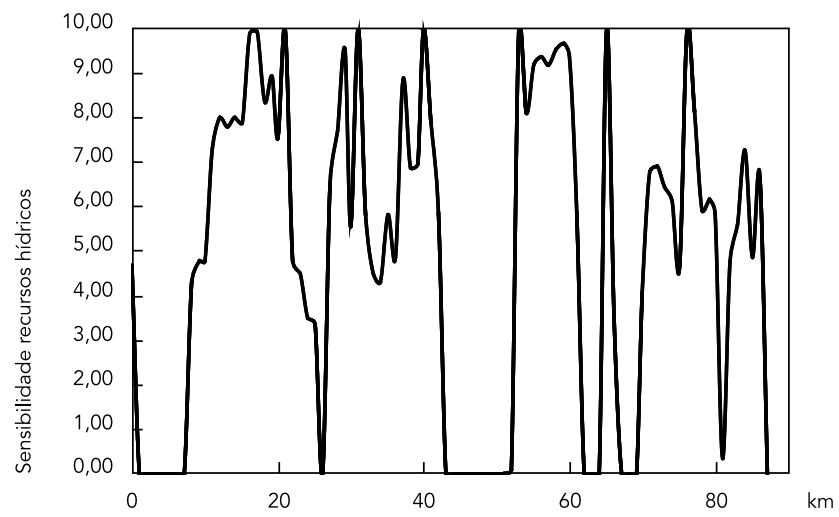

1b) Sensibilidade e vegetação

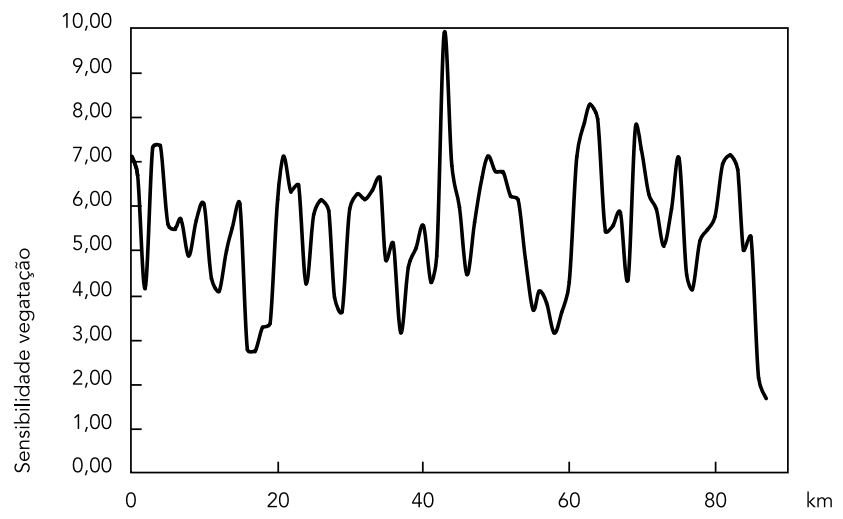

1d) Sensibilidade e ocupação humana

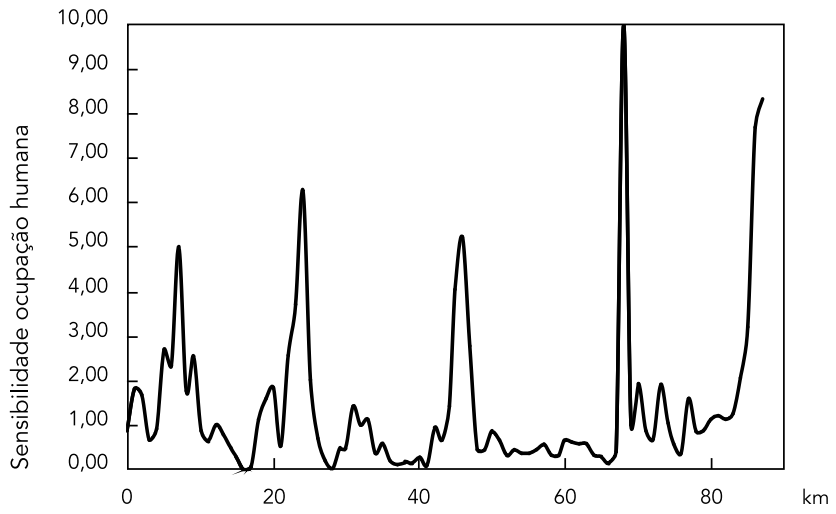

de concentração de população: municípios de Três Cachoeiras (km 24), Terra de Areia (km 46) e Osório (km 86 a 88); aldeia indígena de Campo Bonito (km 8) e o Túnel (Km 69).

\section{Índice de vulnerabilidade ambiental, social e viária}

A Figura 3 apresenta o gráfico do índice de vulnerabilidade ambiental, social e viária na área de influência da rodovia, obtido com base nos resultados da análise de sensibilidade dos recursos hídricos, da vegetação, da ocupação humana e do risco potencial da via, a cada trecho analisado. Os pontos acima da linha horizontal (valor 7,5) são considerados de elevada vulnerabilidade, o que significa que esses quilômetros apresentam uma maior propensão para a ocorrência de acidentes ou uma maior sensibilidade ambiental e social a produtos perigosos transportados ao longo da rodovia. Na Figura 3, podem ser observados os elementos responsáveis pelos valores de vulnerabilidade (por exemplo, pontes sobre rios, lagoas, área de proteção ambiental, áreas de população).

\section{Trechos críticos}

Valendo-se dos índices de vulnerabilidade em cada trecho analisado, foi construído o mapa da rodovia apresentando os resultados da avaliação de vulnerabilidade realizada (Figura 4). 
2a) Risco viário

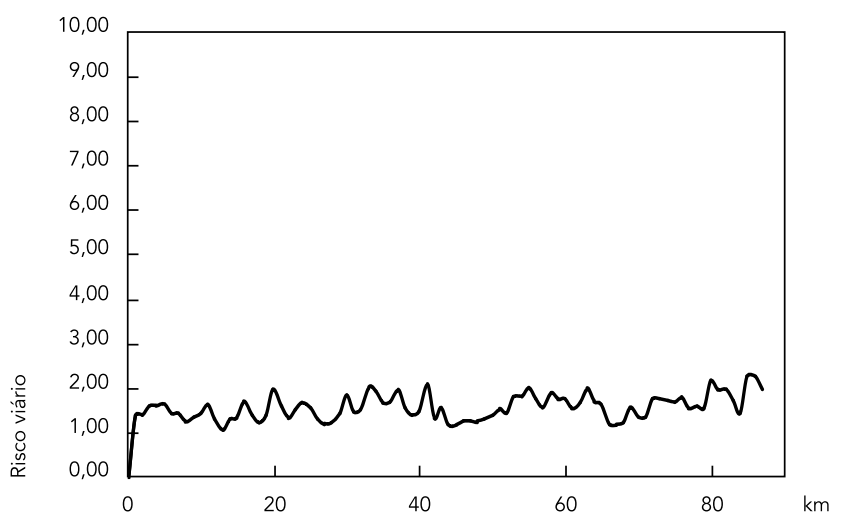

2c) Sensibilidade e recursos hídricos

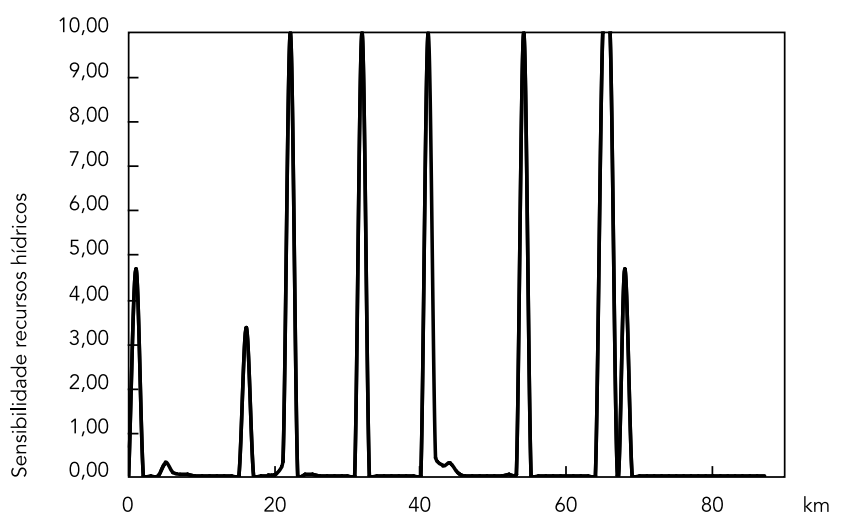

2b) Sensibilidade e vegetação

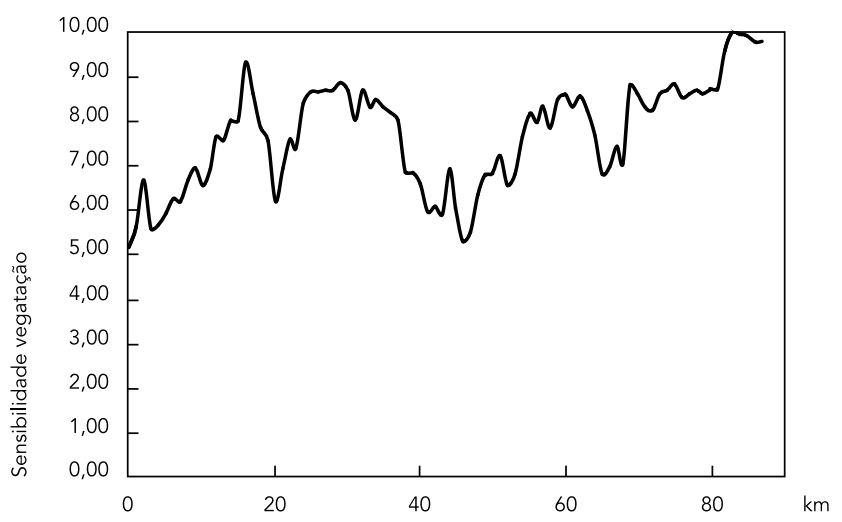

2d) Sensibilidade e ocupação humana

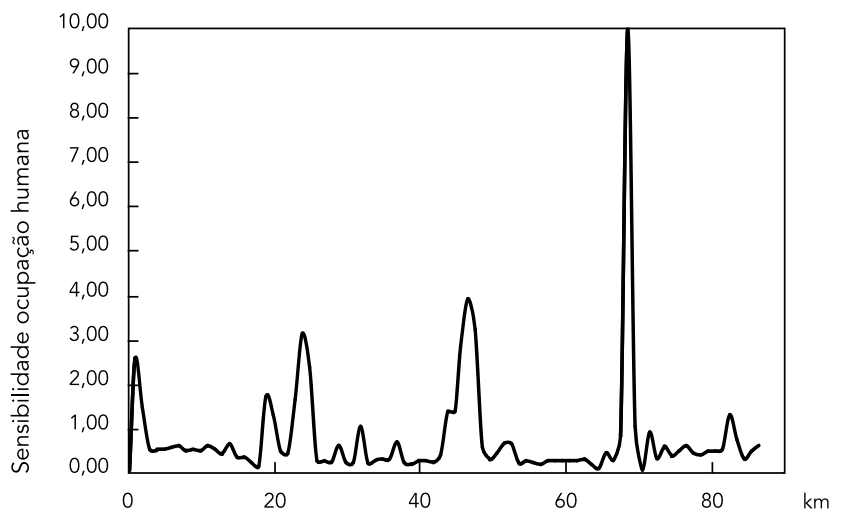

$\mathrm{Na}$ Figura 4, verifica-se que $38 \mathrm{~km}$ da rodovia analisada apresentam vulnerabilidade considerada elevada e $8 \mathrm{~km}$ apresentam vulnerabilidade crítica. Os trechos de vulnerabilidade crítica estão vinculados à presença de pontes sobre rios, pontos de captação para o abastecimento urbano de água, o trecho do túnel e o que compreende a APA Municipal de Osório e zona urbana de Osório. Esses trechos merecem particular atenção no estabelecimento de medidas preventivas e corretivas, além de ações especiais para pronta intervenção saneadora. Outros trechos de elevada vulnerabilidade que devem ser destacados correspondem àqueles onde a rodovia margeia áreas do sistema lagunar, a área suburbana de Três Cachoeiras e os trechos de vegetação nativa e floresta ombrófila densa. Resultados semelhantes em relação às áreas de maior vulnerabilidade foram obtidos em estudos da literatura 2,11,13.

Valendo-se dos resultados de análise de vulnerabilidade ambiental e social e da identificação de trechos críticos, é possível criar uma base de dados e gerar um mapa de vulnerabilidade indicando os trechos mais propensos a acidentes e mais sensíveis aos efeitos destes acidentes com produtos perigosos, ao longo da rodovia. Esse mapa pode auxiliar os órgãos responsáveis no gerenciamento de riscos e no atendimento de emergências no trecho, conforme apontado nas pesquisas da literatura $2,11,13,14$. 


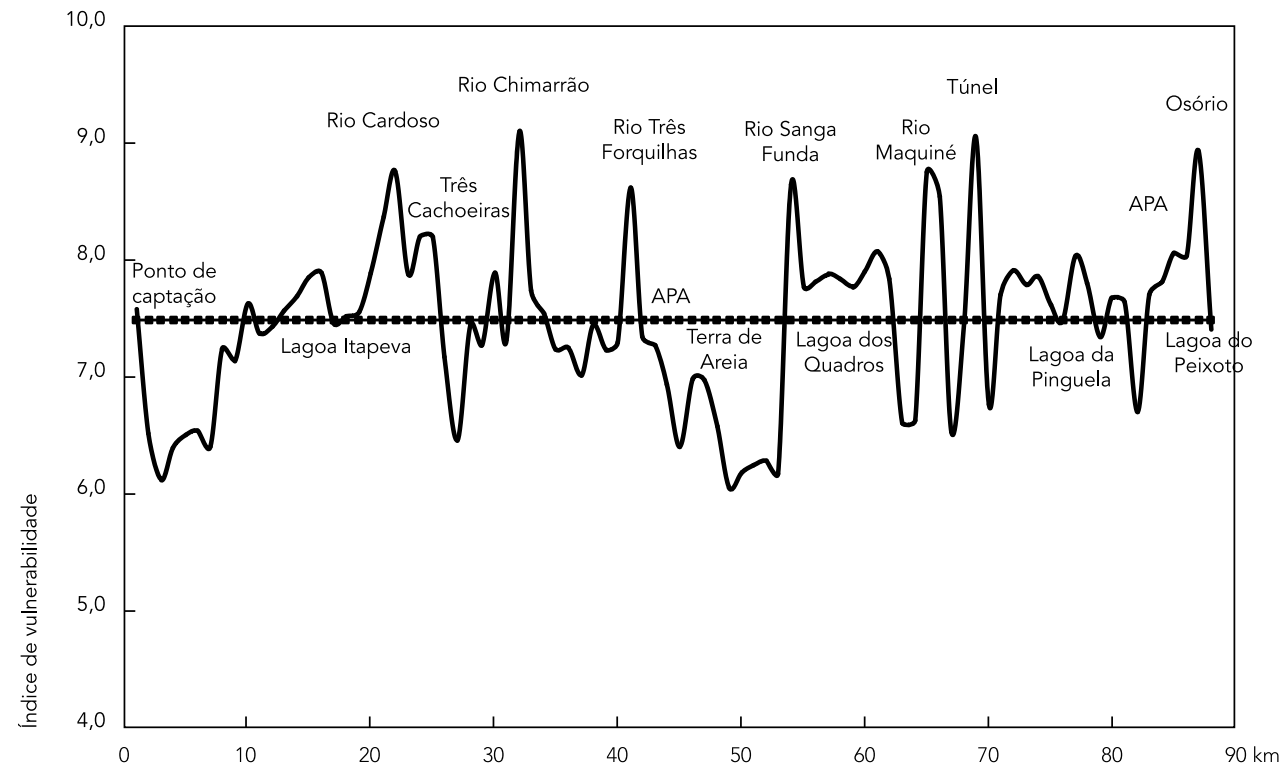

APA: Área de Proteção Ambiental.

\section{Conclusões}

O presente trabalho objetivou a avaliação da vulnerabilidade ambiental e social, associada ao transporte de produtos químicos perigosos e sua aplicação na rodovia BR-101 no trecho de Osório a Torres. O estudo propõe uma abordagem metodológica que integra: (i) avaliação de aspectos físicos de segurança viária da rodovia analisada e (ii) avaliação da sensibilidade de aspectos ambientais e sociais na área de influência da rodovia. Dessa forma, foi determinado um índice de vulnerabilidade para cada trecho analisado da rodovia, o que permitiu identificar trechos críticos, de maior vulnerabilidade, que devem ser priorizados na adoção de ações preventivas para reduzir o risco de acidentes com o transporte de produtos perigosos ou reduzir os efeitos sobre o ambiente e a população.

Com base nos resultados obtidos, sugere-se: (i) a proposição de ações preventivas e a definição de políticas públicas, pelos agentes responsáveis, nos trechos mais vulneráveis à ocorrência de acidentes com produtos perigosos; (ii) a inclusão dos resultados no Plano de Ação Emergencial da rodovia; e (iii) a geração de mapas para o gerenciamento de riscos.
A abordagem utilizada neste trabalho para a avaliação da vulnerabilidade representa um enfoque inédito em relação aos estudos, embora escassos, encontrados na literatura. Uma das principais inovações da abordagem utilizada consiste na estimativa do índice de vulnerabilidade, que foi baseado não somente na sensibilidade dos elementos ambientais (hídricos e vegetação) e sociais (ocupação humana), mas também na análise do RV imposto pela rodovia. A estimação do risco potencial de ocorrência de acidentes foi determinada pelo método de auditoria de segurança viária assistida, aplicado no trecho em estudo.

A avaliação da sensibilidade dos aspectos ambientais e sociais foi baseada em pesquisa de campo, para ponderação da sensibilidade destes aspectos, e na análise de mapas, por meio da aplicação da técnica da quadrícula, que permitiu validar a ponderação estimada pelos pesquisadores, identificando o percentual de cobertura dos aspectos ambientais e sociais na área de influência da rodovia. A integração dessas duas análises permite minimizar a subjetividade da pesquisa qualitativa, gerando resultados mais robustos.

Dessa forma, a abordagem proposta constitui uma ferramenta que pode auxiliar no processo 


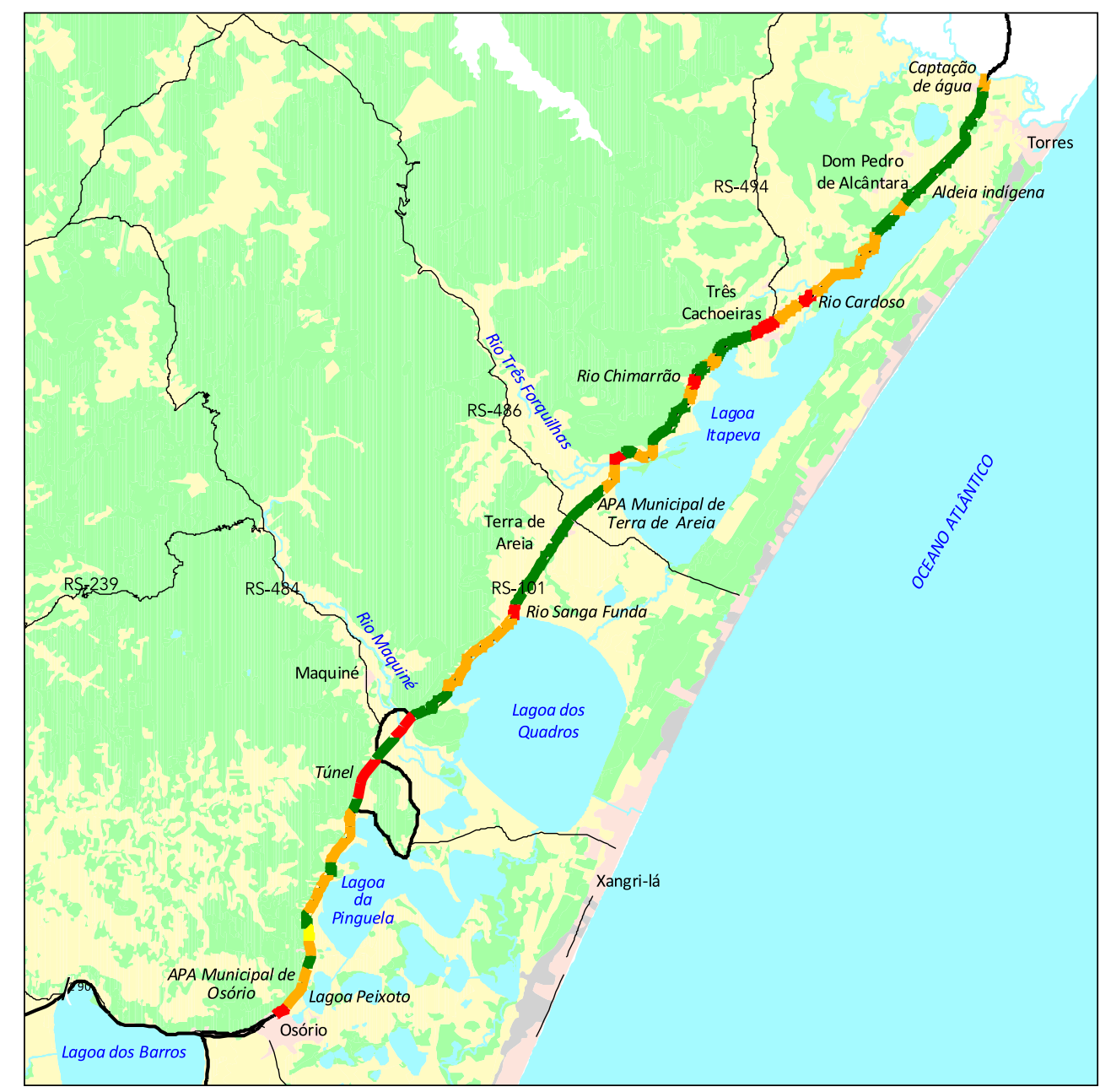

Vulnerabilidade

Baixa

Elevada

Crítica
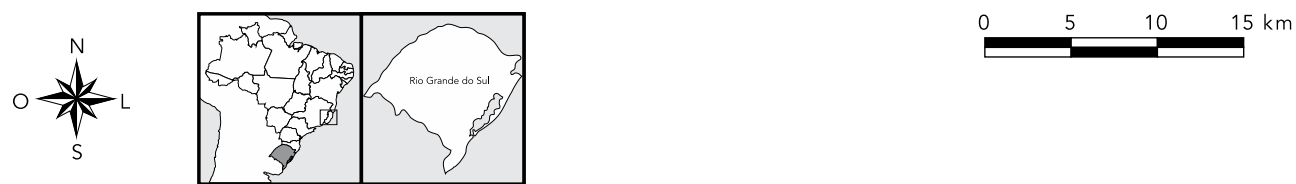

APA: Área de Proteção Ambiental.

de tomada de decisões em operações de emergência, baseando-se na identificação de áreas com maior vulnerabilidade ambiental e social na ocorrência de acidentes com produtos perigosos. O presente estudo permite contribuir com as pesquisas desenvolvidas na área de gerenciamento de riscos no transporte de produtos perigosos. Sugere-se para trabalhos futuros a reapli- cação desta abordagem em outras rodovias para contribuir com a sua validação. 


\section{Colaboradores}

M. A. C. Tinoco contribuiu com a redação do artigo e revisão crítica relevante do conteúdo intelectual. C. T. Nodari contribuiu com a concepção do projeto e aprovação final da versão a ser publicada. K. R. S. Pereira contribuiu com a análise e interpretação dos dados e aprovação final da versão a ser publicada.

\section{Referências}

1. Instituto de Pesquisas Rodoviárias, Departamento Nacional de Infraestrutura de Transportes. Manual para implementação de planos de ação de emergência para atendimento a sinistros envolvendo o transporte rodoviário de produtos perigosos. Rio de Janeiro: Departamento Nacional de Infraestrutura de Transportes; 2005. (Publicação IPR-716).

2. Souza TAR, Hissa LBV, Michael RFM, Morais AML. Análise de multicritério aplicada ao diagnostico do risco ambiental do transporte rodoviário de produtos perigosos: um estudo de caso sobre a BR-381. In: Anais do XIV Simpósio Brasileiro de Sensoriamento Remoto. São José dos Campos: Instituto Nacional de Pesquisas Espaciais; 2009. p. 4465-72.

\section{Agradecimentos}

Os autores agradecem ao Departamento Nacional de Infraestrutura de Transportes (DNIT), pela concessão de bolsas de pesquisa que viabilizaram a realização do trabalho; ao Centro de Pesquisas e Estudos sobre Desastres no Rio Grande do Sul (CEPED/RS) e ao Laboratório de Geoprocessamento (Centro de Ecologia, Instituto de Biociências, Universidade Federal do Rio Grande do Sul), pelo suporte durante a pesquisa realizada.
3. Companhia Ambiental do Estado de São Paulo. Programa de gerenciamento de riscos para administradores de rodovias para o transporte de produtos perigosos. São Paulo: Companhia Ambiental do Estado de São Paulo; 2012.

4. Almeida LVC, Pignatti MG, Espinosa MM. Principais fatores associados à ocorrência de acidentes de trânsito na BR 163, Mato Grosso, Brasil, 2004. Cad Saúde Pública 2009; 25:303-12.

5. Freitas CM, Amorim AE. Vigilância ambiental em saúde de acidentes químicos ampliados no transporte rodoviário de cargas perigosas. Inf Epidemiol SUS 2001; 10:31-42. 
6. Brasil. Decreto no 96.044, de 18 de maio de 1988. Aprova o Regulamento para o Transporte Rodoviário de Produtos Perigosos e dá outras providências. Diário Oficial da União 1988; 19 mai.

7. Agência Nacional de Transporte Terrestre. Resolução no 3.886, de 6 de setembro de 2012. Altera a Resolução ANTT no 3.665, de 4 de maio de 2011, que atualiza o Regulamento para o Transporte Rodoviário de Produtos Perisosos. Diário Oficial da União 2012; 12 set.

8. Agência Nacional de Transporte Terrestre. Resolução no 3.665 , de 4 de maio de 2011. Atualiza o Regulamento para o Transporte Rodoviário de Produtos Perisosos. Diário Oficial da União 2012; 13 mai.

9. Agência Nacional de Transporte Terrestre. Resolução no 420, de 12 de fevereiro de 2004. Aprovas as Instruções Complementares ao Regulamento do Transporte Terrestre de Produtos Perisosos. Diário Oficial da União 2004; 31 mai.

10. Li F, Bi J, Huang L, Qu C, Yang J, Bu Q. Mapping humam vulnerability to chemical acidentes in the vicinity of chemical industry parks. J Hazard Mater 2010; 179:500-6.

11. Bubbico R, Di Cave S, Mazzarotta B. Risk analysis for road and rail transport of hazardous materials: a GIS approach. J Loss Prev Process Ind 2004; 17:483-8.

12. Martínez-Alegría R, Ordóñez C, Taboada J. A conceptual model for analyzing the risks involved in the transportation of hazardous goods: implementation in a geographic information system. Hum Ecol Risk Assess 2003; 9:857-73.

13. Pedro FG, Costa DC. Vulnerabilidade e gravidade ambiental devido a acidentes com transporte rodoviário de combustíveis no Município de Campinas - SP. Revista Brasileira de Cartografia 2009; 61:311-20.

14. Cordeiro FG, Bezerra BS, Peixoto ASP. Methodological aspects for modelling environmental risk of road accidents with transportation of hazardous materials. International Co-operation on Theories and Concepts in Traffic Safety 2012; 1:1-11.
15. Melo CF. Gerenciamento de risco no setor de transporte de produto perigoso no Estado de Santa Catarina: análise no setor de transporte de cargas perigosas [Monografia de Especialização] Tubarão: Universidade do Sul de Santa Catarina; 2010.

16. Nodari TC. Método de avaliação da segurança potencial de segmentos rodoviários rurais de pista simples [Tese de Doutorado]. Porto Alegre: Universidade Federal do Rio Grande do Sul; 2003.

17. Brasil. Lei no 9.985, de 18 de julho de 2000. Regulamenta o art. 225, § 1ㅇ, incisos I, II, III e VII da Constituição Federal, institui o Sistema Nacional de Unidades de Conservação da Natureza e dá outras providências. Diário Oficial da União 2000; 19 jul.

18. Instituto Brasileiro de Geografia e Estatística. Manual técnico da vegetação brasileira. Sistema fitogeográfico. Inventário das formações florestais e campestres. Técnicas e manejo de coleções botânicas. Procedimentos para mapeamentos. 2a Ed. Rio de Janeiro: Instituto Brasileiro de Geografia e Estatística; 2012. Manuais Técnicos em Geociências, 1).

19. Conselho Nacional do Meio Ambiente. Resolução no 357, de 17 de março de 2005. Diário Oficial da União 2005; 18 mar.

20. American Association of State Highway and Transportation Officials. Highway safety manual. Washington DC: American Association of State Highway and Transportation Officials; 2010.

21. Austroads. Road safety audit. Sydney: Austroads National Office; 1994.

22. Ogden K. Safer roads: a guide to road safety engineering. Burlington: Ashgate; 1996.

23. Noy YI, Karwowski W. Human factors in litigation. London: CRC Press; 2005. 


\section{Abstract}

This study aimed to assess the environmental and social vulnerability and identify critical highway stretches for accidents involving transportation of hazardous products on the BR-101 highway between the cities of Osório and Torres in Rio Grande do Sul State, Brazil. The study's approach consisted of a multiple-criteria analysis combining highway safety analysis and environmental and social vulnerability analysis in the occurrence of accidents with hazardous products, plus cartographic analysis techniques. Thirty-eight kilometers of the highway showed high vulnerability, of which 8 kilometers with critical vulnerability, associated with bridges over rivers, water uptake points, a tunnel, environmental preservation areas, and an urban area. These stretches should be prioritized when developing action plans for accident mitigation and development of public policies for this highway. This proved to be an unprecedented approach when compared to existing studies and a potentially useful tool for decision-making in emergency operations.

Vulnerability Analysis; Risk Zone; Traffic Accidents; Hazardous Substances

\section{Resumen}

El presente estudio tiene por objetivo evaluar la vulnerabilidad ambiental social e identificar puntos críticos en la ocurrencia de accidentes, con el transporte de productos peligrosos, en la BR-101 entre los municipios de Osório y Torres, Río Grande do Sul, Brasil. El enfoque propuesta que se aplica en este estudio consiste en un análisis multicriterio que integra metodologías de análisis de seguridad viaria, análisis de vulnerabilidad ambiental y social en la ocurrencia de accidentes con productos peligrosos y técnicas de análisis de mapas. Los resultados señalaron que 38 kilómetros de la autopista presentan vulnerabilidad elevada, existiendo 8 kilómetros con vulnerabilidad crítica, vinculados a la presencia de puentes sobre ríos, puntos de captación de agua, túnel, áreas de preservación ambiental y zona urbana. Esos puntos deben ser priorizados en el desarrollo de planes de acción para la mitigación de accidentes y definición de políticas públicas en la autopista en estudio. El enfoque utilizado constituye un enfoque inédito, en relación con los estudios existentes, y una herramienta que puede auxiliar en el proceso de toma de decisiones en operaciones de emergencia.

Análisis de Vulnerabilidad; Zona de Riesgo;

Accidentes de Tránsito; Sustancias Peligrosas
Recebido em 13/Jul/2015

Versão final reapresentada em 14/Dez/2015

Aprovado em 05/Jan/2016 